\title{
ARTICLE Bile duct ligation enhances AZT CNS toxicity partly by impairing the expression and function of BCRP in rat brain
}

Yuan-yuan Qin ${ }^{1}$, Ping $\mathrm{Xu}^{1}$, Tong $\mathrm{Wu}^{1}{ }^{1}$, Chao-qun Qian ${ }^{1}$, Yi-lin Fan ${ }^{1}$, Dong-hao Gen ${ }^{1}$, Liang Zhu ${ }^{1}$, Wei-min Kong ${ }^{1}$, Han-yu Yang ${ }^{1}$, Feng $\mathrm{Xu}^{1}$, Yi-ting Yang ${ }^{1}$, Li Liu ${ }^{1}$ and Xiao-dong Liu ${ }^{1}$

Breast cancer resistance protein (BCRP) is one of ATP-binding cassette (ABC) transporters in brain microvessel endothelial cells that transport their substrates from brain to blood, thus limiting substrates to crossing into brain through blood-brain barrier. Our previous works show that bile duct ligation (BDL) impairs expression and function of brain BCRP in rats. Since zidovudine (AZT) is $B C R P$ substrate, we investigated whether impaired expression and function of BCRP increased brain distribution and toxicity of AZT in BDL-D7 rats. After administration of AZT (10 mg/kg, i.v.), BDL markedly increased brain AZT concentrations, compared with shamoperated (SO) rats. The ratio of AZT brain-to-plasma area under concentration curve (AUC) in BDL rats was increased to 1.6-folds of SO rats. After treatment with AZT (100 mg/kg every day, i.v.) for 7 days, BDL significantly impaired cognitive functions compared with SO rats, evidenced by the significantly decreased percentage of alternation in Y-maze test and prolonged escaped latency in two-way passive avoidance trial. Furthermore, AZT treatment caused significant decrease in copies of mitochondrial DNA and mitochondrial membrane potential in hippocampus of BDL rats. Moreover, AZT treatment caused a significant decrease of cortex microtubule-associated protein 2 and hippocampus synaptophysin levels in BDL rats. AZT-induced CNS adverse alterations in BDL rats were not observed in SO rats treated with AZT. In conclusion, BDL decreases the function and expression of brain BCRP in rats, leading to increased brain distribution of AZT, which in turn enhances AZT CNS toxicity, leading to mitochondrial dysfunction, neuronal damage, and ultimately cognitive dysfunction.

Keywords: zidovudine; liver failure; breast cancer resistance protein; blood-brain barrier; pharmacokinetics; drug distribution; neurotoxicity; cognitive functions

Acta Pharmacologica Sinica (2020) 41:181-191; https://doi.org/10.1038/s41401-019-0242-8

\section{INTRODUCTION}

Zidovudine (AZT) is often used in the treatment of AIDS as an integral component of highly active antiretroviral therapy. A typical nucleoside/nucleotide reverse transcriptase inhibitor, AZT, undergoes intracellular triphosphorylation and inhibits viral replication by impeding RNA-dependent DNA polymerase. AZT is also used in the treatment of some cancers that also express telomerase [1]. However, accumulating clinical trials have demonstrated that AZT is associated with serious adverse effects [2, 3]. The common adverse effects of AZT therapy include hematological effects (such as anemia and neutropenia), cardiomyopathy, hepatotoxicity, lactic acidosis, myopathy, and neuropathy. These adverse effects of AZT are mainly related to its ability to inhibit telomerase [4] or interfere with mitochondrial function in its target organs $[5,6]$. Although AZT is associated with fetal central nervous system (CNS) toxicity in women receiving treatment during pregnancy [2], relative to those on the peripheral nervous system, the adverse effects of AZT on the CNS are minimal or uncommon; this lack of CNS effect is attributed to low exposure in the brain [7] due to the existence of a blood-brain barrier (BBB).

BBB, an interface for communication between the CNS and blood circulation, consists of brain tight junctions and microvessel endothelial cells connected to the junctional complex by adherent junctions. It plays an important role in limiting toxin entrance into the brain and in extruding toxins out of the brain. Brain microvessel endothelial cells highly express some ATP-binding cassette $(A B C)$ transporters, including P-glycoprotein (P-GP), multidrug resistance-associated protein families (MRPs), and breast cancer resistance protein (BCRP). The $A B C$ transporters mediate transport of their substrates from the brain to the blood, thus limiting the substrates crossing into brain tissues through the BBB. AZT is the substrate of BCRP $[8,9]$, which explains its characteristics of low permeability $[10,11]$. Although the roles of $B C R P$ in the brain distribution of AZT have not been confirmed in Abcg2 $2^{-1-}$ mice [12], low mitochondrial toxicity in the fetal brain after chronic in utero administration of AZT is attributed to low accumulation of AZT in tissues that is at least partly due to BCRP overexpression [13]. We previously reported that liver failure induced by thioacetamide or bile-duct ligation (BDL) impaired the function and expression of BCRP in the BBB of animals, leading to increased distribution of its substrates in the brain [14-16]. Moreover, AZT itself induces liver failure [2]. These results indicate that impairment of BCRP function in the BBB of liver failure rats may increase the brain distribution of AZT, leading to enhancement of CNS toxicity, although the CNS toxicity of AZT is minimal under normal conditions.

${ }^{1}$ Center of Drug Metabolism and Pharmacokinetics, School of Pharmacy, China Pharmaceutical University, Nanjing 210009, China

Correspondence: Li Liu (liulee@cpu.edu.cn) or Xiao-dong Liu (xdliu@cpu.edu.cn)

Received: 29 December 2018 Revised: 19 April 2019 Accepted: 25 April 2019

Published online: 29 May 2019 
The aims of this study were to investigate (1) whether the decreases in the function and expression of BCRP in the BBB of rats with liver failure induced by $B D L$ affect the brain distribution of AZT and (2) whether the increase in the brain distribution of AZT enhances CNS toxicity. The CNS toxicity of AZT was evaluated by assessment of cognitive function impairment, mitochondrial DNA (mtDNA) levels, mitochondrial membrane potential, and microtubule-associated protein 2 (MAP2) and synaptophysin expression in the cortex and hippocampus of rats. The cortex and hippocampus are considered to be related to cognition and learning memory [17-19], which can be easily affected or traumatized by any CNS-toxic impact to the brain. The cortex and hippocampus of rats served as the targeted regions in the study. Schematic illustration of the experiments and group assignments of the study is shown in Scheme 1.

\section{MATERIALS AND METHODS}

Chemicals and materials

AZT, prazosin hydrochloride, and novobiocin were purchased from the National Institutes for Food and Drug Control (Beijing, China). Fluorescein sodium was obtained from Aladdin Co., Ltd (Shanghai, China). A Y-maze and a two-way shuttle box were purchased from Beijing ZS Dichuang Co., Ltd (Beijing, China). A mitochondrial isolation kit was purchased from Beyotime Institute of Biotechnology (Shanghai, China). A GeneJET genomic DNA purification kit was purchased from Thermo Fisher Scientific Inc. (Waltham, MA, USA). Synaptophysin (ab32127) and MAP2 (ab32454) antibodies, AF488 goat anti-rabbit secondary antibodies, and DAPI were purchased from Abcam Company (Cambridge, UK). BCRP (42078 S) and $\beta$-actin (4970 S) antibodies and HRP-conjugated secondary antibodies were purchased from Cell Signaling Technology (Boston, MA, USA). Madin-Darby canine kidney II (MDCKII-WT) cells and stably BCRP-expressing MDCKII (MDCKII-BCRP) cells were purchased from Biowit Technologies, Ltd (Shenzhen, China). A human cerebral microvessel endothelial cell line (HCMEC/D3) was purchased from JENNIO Biological Technology, Ltd (Guangzhou, China). Opti-MEM and Lipofectamine 3000 were purchased from Invitrogen (Waltham, CA, USA). All the other reagents were of analytical grades and were commercially available.

Establishment of liver failure rats by BDL

Male Sprague-Dawley (SD) rats weighing $220 \mathrm{~g}$ were provided by Super-B\&K Experimental Animal Co., Ltd (Shanghai, China). The animals had free access to food and water under controlled environmental conditions (temperature $24 \pm 2{ }^{\circ} \mathrm{C}$, humidity $50 \% \pm$ $5 \%, 12 \mathrm{~h}$ light/dark cycle). Following 7 days of acclimation, surgical procedures were performed to establish BDL-induced liver failure rats according to a method described previously [16]. Shamoperated (SO) rats were treated in the same manner without BDL. On day 7 following surgery, the animals were used for the following experiments. The animal experiments were carried out according to the Institutional Guidelines for the Care and Use of Laboratory Animals and were authorized by the Animal Ethics Committee of China Pharmaceutical University (No. CPU-PCPK1621010279).

Brain distributions of prazosin and fluorescein sodium in BDLinduced liver failure rats

The distributions of prazosin and fluorescein sodium were analyzed to assess BCRP function and BBB integrity, respectively. Briefly, on day 7 following surgery, animals fasted overnight were sacrificed under light diethyl ether $45 \mathrm{~min}$ after injection of mixtures consisting of prazosin ( $1 \mathrm{mg} / \mathrm{kg}$, i.v.) and fluorescein sodium ( $2 \mathrm{mg} / \mathrm{kg}$, i.v.). Blood, cortex, and hippocampus samples were then obtained. The plasma and serum were used for biochemical and drug analysis, while the brain tissues were used for drug analysis and Western blotting. The spleens and livers were also weighed. The concentrations of prazosin in plasma and brain tissues were measured by high-performance liquid chromatography [15]. The concentrations of fluorescein sodium in plasma and brain tissues were measured by liquid chromatography-mass spectrometry [20]. Serum biochemical parameters such as aspartate transaminase (AST), alanine transaminase (ALT), and alkaline phosphatase (ALP) activity and bile acid (TBA) and total bilirubin (TBIL) levels were measured using corresponding commercial assay kits (Jiancheng Bioengineering Institute, Nanjing, China).

Brain distribution kinetics of AZT in BDL-induced liver failure rats On day 7 following surgery, animals (4 rats for each time point) fasted overnight were sacrificed under light diethyl ether $5,15,30$, $45,60,120$, and $180 \mathrm{~min}$ after administration of AZT $(10 \mathrm{mg} / \mathrm{kg}$, i.v.). Subsequently, blood samples and brain tissues were obtained. Brain homogenates, supernatants, and pellet samples were prepared according to a method described previously [21]. The plasma, brain homogenates, supernatants, and pellet samples were stored at $-80^{\circ} \mathrm{C}$ until analysis. The AZT analysis process is available in the Supplementary Information.

\section{In situ brain perfusion}

In situ brain perfusion was used to investigate the contribution of brain BCRP to the brain distribution of AZT in normal rats according to a procedure described previously $[22,23]$. The perfusate buffer consisted of bicarbonate-buffered physiological solution (in mM: $128 \mathrm{NaCl}, 24 \mathrm{NaHCO}_{3}, 4.2 \mathrm{KCl}, 2.4 \mathrm{NaH}_{2} \mathrm{PO}_{4}, 1.5$ $\mathrm{CaCl}_{2}, 0.9 \mathrm{MgCl}_{2}$, and 9 D-glucose) containing AZT (25 $\left.\mu \mathrm{M}\right)$ or AZT $(25 \mu \mathrm{M})+$ novobiocin $(200 \mu \mathrm{M})$ and was oxygenated with a mixture of $95 \% \mathrm{O}_{2}$ and $5 \% \mathrm{CO}_{2}$ and heated at $37^{\circ} \mathrm{C}$ in a water bath. After perfusion at a flow rate of $2.5 \mathrm{~mL} / \mathrm{min}$ for $2 \mathrm{~min}$, the perfusion was terminated, and the animals were sacrificed. Right hippocampus and brain cortex samples were immediately obtained, and the AZT levels $\left(C_{b}\right)$ in brain tissues were measured by LC-MS/MS. The apparent unidirectional influx constants $\left(K_{\mathrm{in}}\right)$ were calculated with the equation $K_{\text {in }}=C_{\mathrm{b}} /\left(T \times C_{\mathrm{pf}}\right)$, where $C_{\mathrm{pf}}$ and $T$ are the AZT concentrations in the perfusate and the perfusion time, respectively.

AZT treatment and adverse effects on the CNS

On day 7 following surgery, the SO and BDL rats were randomly separated into control rats and rats treated with AZT to form four groups: SO rats (control group), SO rats treated with AZT (SO + AZT group), BDL rats (control group), and BDL rats treated with AZT (BDL + AZT group). Control rats received only vehicle, and rats treated with AZT intravenously received AZT $(100 \mathrm{mg} / \mathrm{kg}$ every day) for another 7 days. The AZT dose was taken from a previous report [24]. During AZT treatment, cognitive function and exploratory ability were evaluated using a two-way passive avoidance test and a Y-maze test, respectively.

The Y-maze test was performed to assess spatial memory according to a method described previously [25]. A Y-shape design with an arm length of $47 \mathrm{~cm}$ was used. On day 5 of AZT treatment, rats were placed at the end of the starting arm and allowed to explore the two other arms of the maze for $5 \mathrm{~min}$. The number of times each rat explored the novel arms relative to the total number of times the rat visited the three arms and the percentage of novel arm entry during the test phase were recorded.

A two-way passive avoidance test was performed to study learning and working memory status in the animals using a twoway shuttle box according to a method described previously [26]. Before the beginning of the first training session, each animal could ambulate freely in the shuttle box for $2 \mathrm{~min}$ to become familiar with the training setup. On day 4 of AZT treatment, the first training session began; the animals were subjected to training 

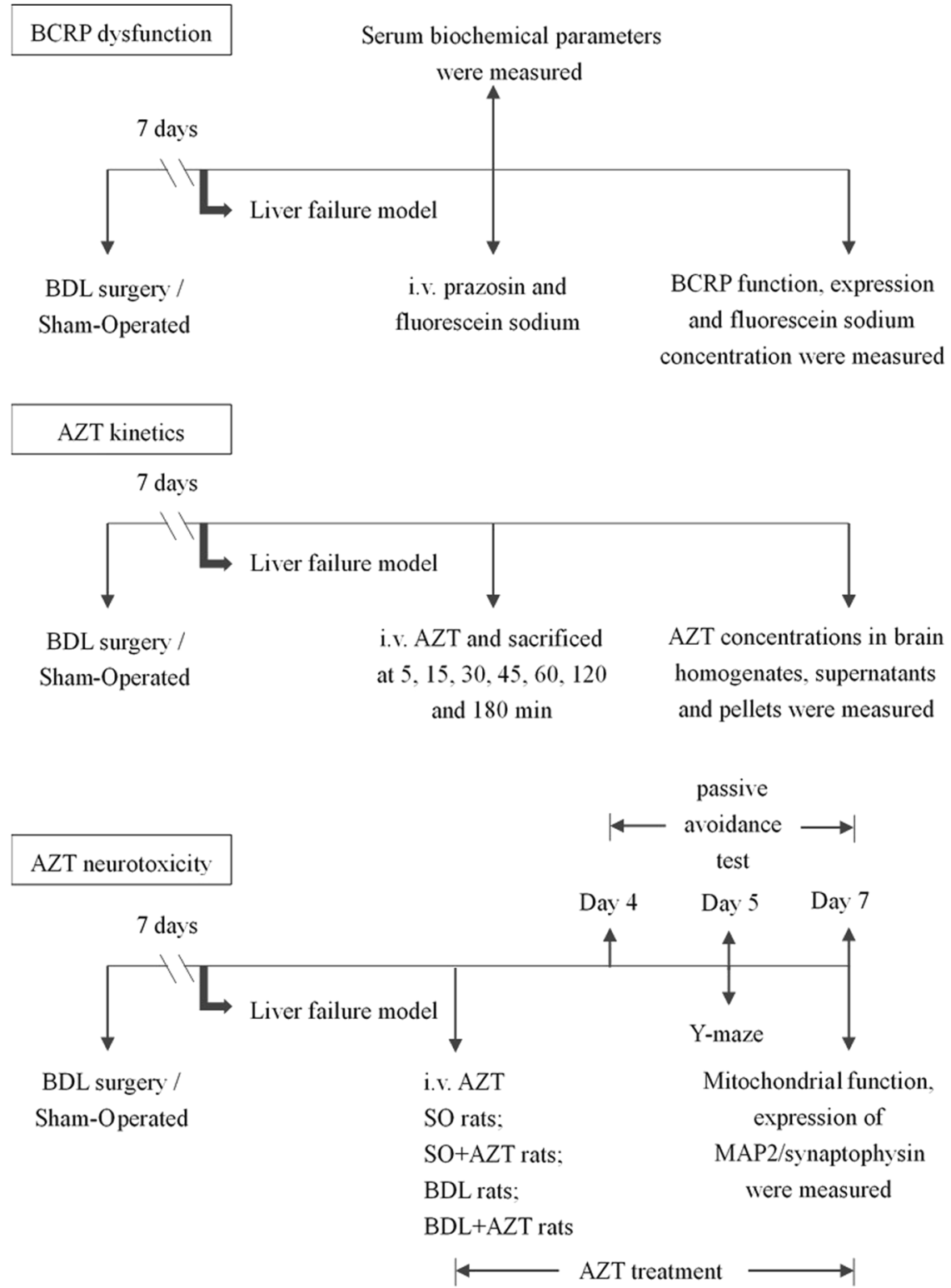

Scheme 1 Schematic illustration of the experiments and group assignments of the study

sessions for 4 days (a session involved 20 trials, and one session was conducted per day). The fourth session data were used to evaluate the long-term retention of the learned response. The apparatus was computer controlled, and the software was programmed exclusively in a programming language for the shuttle-box experiment.

At $24 \mathrm{~h}$ after the last dose, the rats were sacrificed under light diethyl ether. The brain tissues were quickly obtained and used for assessment of mitochondrial membrane potential, synaptophysin and MAP2 expression and mtDNA levels. Another batch of rats was used for immunofluorescence staining of brain tissues.

Measurement of mitochondrial membrane potential Mitochondria from fresh brain tissues were isolated using a tissue mitochondrial isolation kit according to the manufacturer's instructions. The protein concentrations of the mitochondria were determined using a BCA assay kit. Mitochondrial membrane potential was immediately assessed using a mitochondrial membrane potential assay kit with JC-1. Briefly, each mitochondrial suspension was diluted with mitochondria storage buffer. The samples were incubated in $\mathrm{JC}-1$ solution at $37^{\circ} \mathrm{C}$ for 5 min; then, fluorescence was measured at $490 \mathrm{~nm}$ excitation and $530 \mathrm{~nm}$ emission for monomers and at $525 \mathrm{~nm}$ excitation and 590 $\mathrm{nm}$ emission for J-aggregates. The membrane potential is expressed as the ratio of the fluorescence units of J-aggregates to those of monomers.

Extraction and measurement of mitochondrial DNA in the brain The copy numbers of mtDNA (ND1, ND2, and ND4) were measured using $\mathrm{qPCR}$. Genomic DNA was purified from rat cortex and hippocampus using a GeneJET genomic DNA purification kit according to the manufacturer's instructions. QPCR was performed using THUNDERBIRD SYBR qPCR Mix (Toyobo Co., Ltd, Osaka, Japan) on a Roche LightCycler 96 (Penzberg, Germany) for relative quantification. The primer sequences of the target genes were as follows: ND1: forward 5'-AATACGCCGCAGGACCATTC-3', reverse 5'-G GGGTAGGATGCTCGGATTC-3'; ND2: forward 5'-CCTCATAGGGCCTGT AATCACT-3', reverse: 5'-GCTGCTTCAGTTGATCGTGG-3'; ND4: forward 
5'-AACGAATCCACAGCCGAACT-3', reverse 5'-AGGGGTGGTAGTGCTA GGTT-3'; and GAPDH: forward 5'-ATTGTCAGCAATGCATCCTG-3', reverse $5^{\prime}$-ATGGACTGTGGTCATGAGCC-3'. The levels of the mtDNAs were normalized to those of GAPDH $\left(2^{-\Delta \mathrm{Ct}}\right.$ method).

\section{Immunofluorescence staining of tissues}

Slides of paraffin-embedded tissue sections from the cortex and hippocampus of BDL and SO rats were prepared for immunofluorescence staining. Briefly, sections $(8 \mu \mathrm{m})$ were heated at $65^{\circ} \mathrm{C}$ for $2 \mathrm{~h}$, deparaffinized in dimethylbenzene, and rehydrated with consecutive $100 \%, 95 \%, 90 \%, 80 \%$, and $70 \%$ ethanol washes. The sections were blocked with $10 \%$ normal goat serum in phosphatebuffered saline (PBS). A rabbit monoclonal antibody to synaptophysin (1:500 dilution) or a rabbit polyclonal antibody to MAP2 (1:500 dilution) was used, and the sections were incubated overnight at $4{ }^{\circ} \mathrm{C}$. After washing the slides with PBS, the sections were incubated with an AF488 goat anti-rabbit secondary antibody (1:1000 dilution). The sections were then washed with PBS, and DAPI was used to stain the nuclei for $5 \mathrm{~min}$. Immunofluorescence images of the slides were obtained with a confocal laser scanning microscope (LSM700; Zeiss, Germany). The staining densities were quantified using an image analysis software program.

\section{Western blot analysis}

Brain tissues were homogenized in RIPA lysis buffer. The protein concentrations were determined using a BCA assay kit, and $40 \mu \mathrm{g}$ of protein was fractionated by sodium dodecyl sulfate-polyacrylamide gel electrophoresis. The separated proteins were transferred to polyvinylidene fluoride membranes. After blocking with $5 \%$ nonfat milk for $1 \mathrm{~h}$, the membranes were incubated overnight with rabbit polyclonal anti-BCRP (1:1000 dilution), anti-synaptophysin (1:50000 dilution), anti-MAP2 (1:2000 dilution), and anti- $\beta$-Actin (1:4000 dilution). After rigorously washing, the membranes were incubated with appropriate HRP-conjugated secondary antibodies (1:5000 dilution). The immunoreactivity was detected using SuperSignal West Femto Chemiluminescent Substrate (Thermo Fisher Scientific Inc., Waltham, MA, USA) on a gel imaging system (Tanon 5200 Multi; Tanon Technology, Co., Ltd, Shanghai, China).

Cell culture and uptake of AZT

Cell uptake of AZT was documented in MDCKII-WT, MDCKIIBCRP, and HCMEC/D3 cells. MDCKII-WT and MDCKII-BCRP cells were seeded in 24-well plates at a density of $2.0 \times 10^{5}$ cells per well in DMEM. BCRP expression in MDCKII-BCRP cells was confirmed using Western blot analysis. HCMEC/D3 cells were seeded in 24-well plates at $2.0 \times 10^{5}$ per well in RPMI- 1640 . When they reached confluence, the cells were used for the uptake study. Pre-experiments showed that cell uptake of AZT in MDCKII-BCRP cells plateaued after 90-120 min of incubation and that the uptake was linear, ranging from 0.5 to $2 \mu \mathrm{M}$. Thus, the uptake time and AZT concentration were set to $120 \mathrm{~min}$ and $1.0 \mu \mathrm{M}$, respectively. Briefly, the uptake experiment was initiated by adding $0.5 \mathrm{~mL}$ of HBSS containing AZT $(1 \mu \mathrm{M})$. Following a 120 -min incubation, the uptake reaction was terminated by rinsing three times with ice-cold HBSS. The concentrations of intracellular AZT and the protein concentrations were measured using LC-MS/MS and a BCA protein assay kit, respectively.

The effects of both liver failure rat serum and unconjugated bilirubin on the uptake of AZT in MDCKII-BCRP cells were also measured. The cells were incubated with medium containing $10 \%$ rat serum or unconjugated bilirubin $(0,10$, and $25 \mu \mathrm{M})$. Then, the uptake of AZT in MDCKII-BCRP cells was measured as described above.

Small interfering RNA knockdown

A small interfering RNA (siRNA) knockdown experiment was conducted to assess the role of BCRP in the transport of AZT.
Table 1. Physiological and biochemical parameters of sham-operated (SO) and bile duct ligation (BDL) rats at $d 7$ following surgical operation

\begin{tabular}{lcc}
\hline Parameters & SO rats & BDL rats \\
\hline Body weight $(\mathrm{BW})(\mathrm{g})$ & $223 \pm 18$ & $224 \pm 11$ \\
Liver weight $(\% \mathrm{BW})$ & $3.6 \pm 0.4$ & $4.6 \pm 0.7^{* *}$ \\
Spleen weight $(\% \mathrm{BW})$ & $0.33 \pm 0.05$ & $0.31 \pm 0.08$ \\
ALT $(\mathrm{IU} / \mathrm{L})$ & $21.51 \pm 3.71$ & $42.42 \pm 11.19^{* *}$ \\
AST $(\mathrm{IU} / \mathrm{L})$ & $14.35 \pm 1.83$ & $38.85 \pm 14.13^{* *}$ \\
ALP $(\mathrm{IU} / \mathrm{L})$ & $152.69 \pm 12.22$ & $241.19 \pm 51.89$ \\
Total bilirubin $(\mu \mathrm{M})$ & $9.83 \pm 3.34$ & $140.35 \pm 57.00^{* *}$ \\
Total bile acids $(\mu \mathrm{M})$ & $54.16 \pm 24.73$ & $140.77 \pm 28.50^{* *}$ \\
\hline $\begin{array}{l}\text { Data were expressed as mean } \pm \text { SD of } 8 \text { rats, }{ }^{* *} P<0.01 \text { vs SO rats } \\
\text { ALT alanine transaminase, } A S T \\
\text { phosphatase }\end{array}$ & \multicolumn{2}{l}{ aspartate transaminase, $A L P$ alkaline } \\
\hline
\end{tabular}

HCMEC/D3 cells were seeded in 24-well plates at a density of $2.0 \times$ $10^{4}$ cells per well in RPMI-1640. The next day, the cells were transfected with siRNA for human ABCG2 (GGCAAAUCUUCGU UAUUAGtt) [27] or with a scrambled negative control (GenePharma, Shanghai, China) using Lipofectamine 3000. Following 48 or $60 \mathrm{~h}$ of culture, the protein levels of BCRP and the uptake of AZT in HCMEC/D3 cells were measured as described above.

Data analysis

The results are expressed as the mean \pm standard deviation (SD). Comparisons between two groups were performed using $t$-tests. Comparisons among multiple groups were performed by one-way analysis of variance followed by least significant difference tests. $P<0.05$ was considered to indicate statistical significance.

\section{RESULTS}

Alterations in physiological and biochemical parameters in BDL rats The data for the physiological and biochemical parameters confirmed the establishment of BDL-induced liver failure rats (Table 1). Compared with SO rats, BDL rats showed increased liver weight and increased ALT, AST, ALP, total bile acid, and total bilirubin levels. In particular, the levels of total bilirubin were increased by 14-fold in $\mathrm{BDL}$ rats compared to SO rats. These results indicated that liver failure in rats was successfully induced by bile duct ligation.

Effect of BDL on the function and expression of brain BCRP The brain distribution of prazosin was used to characterize the function of BCRP in the brain (Fig. 1a, b). The results showed that BDL had no effect on the concentrations of prazosin in both the cortex and hippocampus but significantly decreased the concentrations in plasma, leading to 2.3-fold higher brain-to-plasma concentration ratios of prazosin in $\mathrm{BDL}$ rats than in SO rats. Data regarding the leakage of fluorescein sodium showed that BDL did not impair the integrity of the BBB (Fig. 1C, d), suggesting that the increased brainto-plasma concentration ratios of prazosin were partly due to functional impairment of BCRP in the brains of BDL rats.

The expression of BCRP in the rat cortex and hippocampus was detected using Western blot analysis (Fig. 1e, f). Consistent with dysfunction of BCRP, BDL rats showed decreased expression of BCRP protein both in the cortex and hippocampus; the expression of $B C R P$ in the brains of BDL rats was decreased to approximately $60 \%$ of that in the brains of SO rats.

Brain distribution kinetics of $A Z T$ in $B D L$ rats

On day 7 following surgery, the concentrations of AZT in rat plasma, brain homogenates, brain supernatants, and brain pellets 


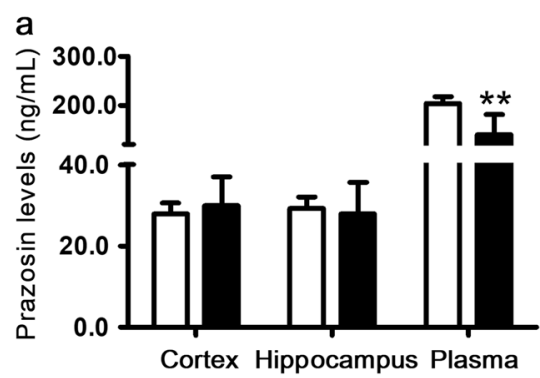

d

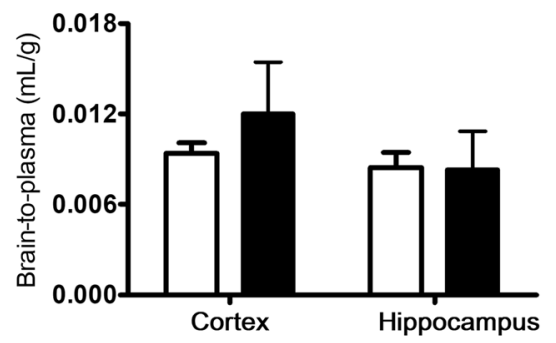

b

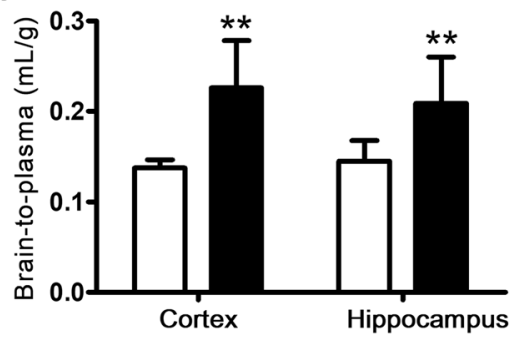

e

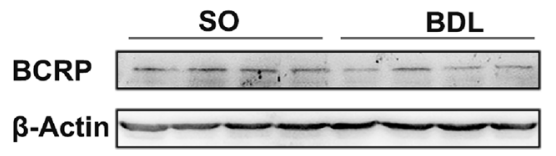

Cortex

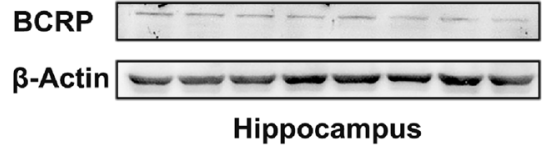

C

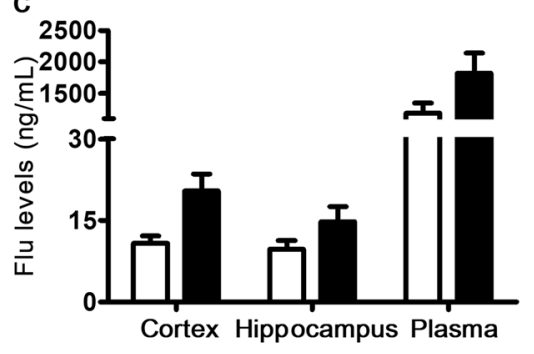

f

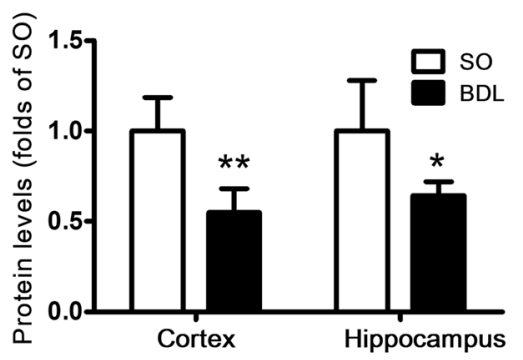

Fig. 1 Effects of BDL on the function and expression of BCRP in the brains of rats. Concentrations of prazosin (a) in the plasma, cortex, and hippocampus in sham-operated (SO) and bile duct ligation (BDL) rats and the brain-to-plasma concentration ratios of prazosin (b) 45 min after administration (1 mg/kg, i.v.). Concentrations of fluorescein sodium (Flu, c) in rat plasma, cortex, and hippocampus and the brain-to-plasma concentration ratios of Flu (d) $45 \mathrm{~min}$ after administration ( $2 \mathrm{mg} / \mathrm{kg}$, i.v.). The data are expressed as the mean \pm SD for eight rats. Expression of BCRP protein in the cortex and hippocampus in SO and BDL rats $(\mathbf{e}, \mathbf{f})$. The data are expressed as the mean $\pm S D$ for four rats. ${ }^{*} P<0.05$, ${ }^{* *} P<0.01$ vs SO rats

were measured after administration of AZT (10 mg/kg, i.v.) (Fig. 2a-d), and corresponding pharmacokinetic parameters were estimated (Table 2). Since the brain concentrations of AZT might be affected by its plasma concentrations, the brain-to-plasma concentration ratios of AZT were also calculated (Fig. 2e-g). The results showed that $B D L$ did not affect the concentration-time curves of AZT in plasma but markedly increased the distribution of AZT in brain homogenates, supernatants, and pellets. The data regarding the brain-to-plasma concentration ratios of AZT demonstrated that the brain-to-plasma ratios in $\mathrm{BDL}$ rats increased with time, indicating that efflux of AZT from the brains of BDL rats was impaired. Compared with SO rats, BDL rats exhibited higher AZT levels in brain homogenates, supernatants, and pellets. When AZT was administered intravenously, it exhibited a slower elimination phase (evidenced by a longer $t_{1 / 2}$ ) in BDL rats than in $S O$ rats. The AUC values for AZT in the BDL group were markedly increased to levels approximately 1.5 -fold higher than those in the SO group. The $t_{1 / 2}$ for brain supernatants was longer than that for homogenates and pellets from SO rats, while the $t_{1 / 2}$ for brain homogenates was longer than that for supernatants and pellets from BDL rats. The AUC for brain homogenates was greater than that for supernatants and pellets from both SO and BDL rats. The drug targeting indexes (DTIs) were estimated with the equation: $\mathrm{DTI}=\left(\mathrm{AUC}_{\text {brain }} / \mathrm{A} \cup C_{\text {plasma }}\right)_{\mathrm{BDL}} /\left(\mathrm{A} \cup C_{\text {brain }} / \mathrm{AUC}_{\text {plasma }}\right)_{\mathrm{SO}}$. It was found that the DTIs for brain homogenates, supernatants, and pellets from $B D L$ rats were 1.66, 1.62, and 1.62, respectively, further confirming that BDL increased the brain distribution of AZT.

Brain uptake of $A Z T$ in normal rats in the presence of a typical BCRP inhibitor (novobiocin) was also assessed to further confirm the roles of brain BCRP in the brain distribution of AZT. The data from the in situ brain perfusion (Fig. 2h) demonstrated that coadministration of novobiocin increased the brain uptake coefficient $\left(K_{\text {in }}\right)$ of AZT to 2.3 -fold of that without novobiocin in the hippocampus of rats. Although the cortex showed a trend toward an increase in $K_{\text {in, }}$ the increase was not significant. These results demonstrated the contribution of $B C R P$ to the brain distribution of AZT, at least in the hippocampus.

Effects of AZT treatment on the cognitive behaviors of experimental rats

The effects of AZT treatment on cognitive behaviors were assessed using a Y-maze and a two-step shuttle box. The data from the Y-maze test showed that BDL markedly decreased the total number of entries. The decrease in BDL rats was further enhanced by AZT treatment, but the difference was not significant. It was also found that administration of AZT significantly decreased the percentage of alternation of arm entrance in BDL rats compared with SO rats. These results suggested that AZT treatment might exacerbate the impairment of the ability to explore new environments caused by BDL. A twostep shuttle box was used to test the status of learning and working memory in rats. The results were consistent with the $Y$ maze results: BDL rats treated with $A Z T$ showed clearly increased escape latencies compared to SO rats (Fig. 3c). The impairment of cognitive function by AZT that was observed in BDL rats did not occur in SO rats.

Effects of AZT treatment on mtDNA expression and mitochondrial membrane potential in the brain

The effects of 7-day AZT administration on the expression of mtDNA (termed ND1, ND2, and ND4) in the cortex and hippocampus of the rat brain were assessed using $q P C R$. The mtDNA copy number was quantified as the ratio of the expression of the target gene to that of a nuclear gene (GAPDH) (Fig. 4a-c). The results showed that AZT markedly decreased the copy numbers of ND1, ND2, and ND4 in the hippocampus of BDL rats. The hippocampal copy numbers of ND1, ND2, and ND4 in BDL rats treated with AZT were $58 \%, 40 \%$, and $54 \%$ lower, respectively, than those in the BDL rats not treated with AZT (Fig. 4). No significant differences were found in the cortex mtDNA copy numbers of the rats due to individual variation. 

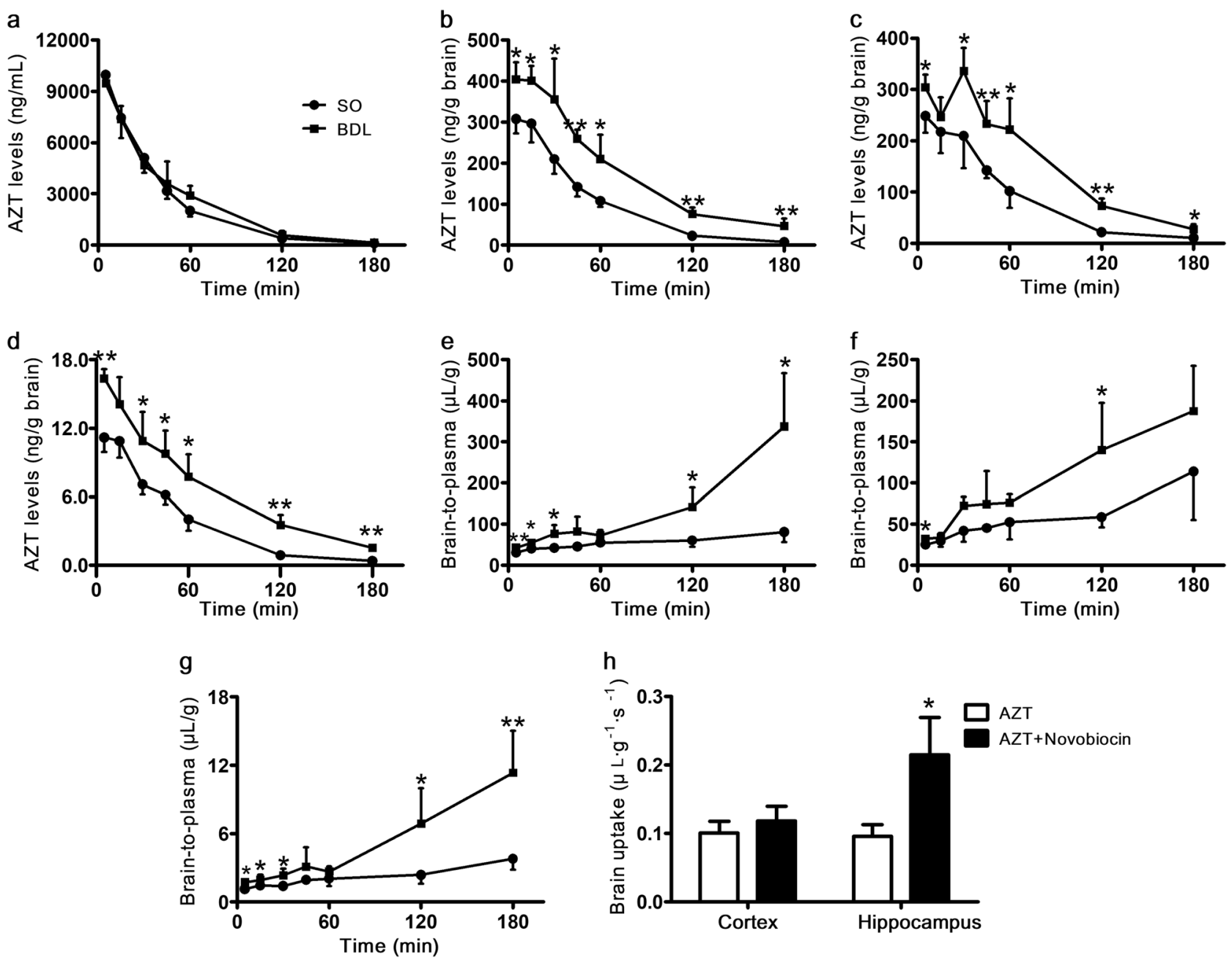

Fig. 2 Effects of BDL on the brain distribution of zidovudine (AZT). AZT concentrations in plasma (a), brain homogenates (b), brain supernatants (c), and brain pellets (d), and the corresponding brain-to-plasma concentration ratios (e, f, $\mathbf{g})$ in sham-operated (SO) and bile duct ligation $(\mathrm{BDL})$ rats. The data are expressed as the mean \pm SD for four rats. ${ }^{*} P<0.05$ and ${ }^{* *} P<0.01$ vs SO rats. Effects of novobiocin on the brain uptake of AZT delivered by in situ perfusion in normal rats (h). The data are expressed as the mean \pm SD for five rats. ${ }^{*} P<0.05$ vs AZT alone

Table 2. Pharmacokinetic parameters of zidovudine in plasma $(\mathrm{PL})$, brain homogenates $(\mathrm{BH})$, brain supernatants $(\mathrm{BS})$, and brain pellets $(\mathrm{BP})$ of sham-operated $(\mathrm{SO})$ and bile duct ligation $(\mathrm{BDL})$ rats following administration of AZT $(10 \mathrm{mg} / \mathrm{kg}$, i.v.)

\begin{tabular}{|c|c|c|c|c|c|c|c|c|}
\hline \multirow[b]{2}{*}{ Parameters } & \multicolumn{4}{|l|}{ SO rats } & \multicolumn{4}{|c|}{$\mathrm{BDL}$ rats } \\
\hline & PL & $\mathrm{BH}$ & BS & $\mathrm{BP}$ & PL & $\mathrm{BH}$ & BS & BP \\
\hline$t_{1 / 2}(\min )$ & 26.28 & 30.79 & 35.78 & 33.61 & 27.63 & 51.41 & 39.54 & 50.82 \\
\hline $\begin{array}{l}\text { AUC (min• } \\
\mu \mathrm{g} / \mathrm{mL} \text { or } \mathrm{g} \text { ) }\end{array}$ & 422.61 & 17.70 & 15.99 & 0.66 & 461.90 & 32.06 & 28.23 & 1.20 \\
\hline $\begin{array}{l}\mathrm{AUC}_{i} / \\
\mathrm{AUC} \mathrm{C}_{\mathrm{PL}}(\mu \mathrm{L} / \\
\mathrm{g})\end{array}$ & 1.00 & 41.88 & 37.84 & 1.56 & 1.00 & 69.41 & 61.12 & 2.60 \\
\hline $\begin{array}{l}\mathrm{CL}(\mathrm{mL} / \mathrm{kg} / \\
\min )\end{array}$ & 23.44 & - & - & - & 21.39 & - & - & - \\
\hline$V_{\mathrm{ss}}(\mathrm{mL} / \mathrm{kg})$ & 822.47 & - & - & - & 870.63 & - & - & - \\
\hline
\end{tabular}

$P L$ plasma, $B H$ brain homogenates, $B S$ brain supernatants, $B P$ brain pellets, $S O$ sham-operated, $B D L$ bile duct ligation, $A U C$ area under concentration curve, $\mathrm{AUC}_{i}(i=\mathrm{PL}, \mathrm{BH}, \mathrm{BS}, \mathrm{BP}) ; V_{\mathrm{ss}}$ apparent volume of distribution
Mitochondrial membrane potential was measured using JC-1 fluorescent dye. The results showed that BDL rats administered AZT exhibited markedly $(P<0.01)$ decreased hippocampal mitochondrial membrane potential compared with non-AZT-treated $\mathrm{BDL}$ rats. The measured hippocampal mitochondrial membrane potential of BDL rats treated with AZT was only approximately $60 \%$ of that of BDL rats not treated with AZT (Fig. 4d), while the mitochondrial membrane potential in the rat cortex was unaltered.

Effects of AZT treatment on MAP2 and synaptophysin in the brain

MAP2 and synaptophysin often serve as neuronal loss and synaptic damage indicators, respectively [24]. The effects of AZT treatment on the expression of MAP2 and synaptophysin were investigated using Western blot and immunofluorescence analyses. The results showed that neither AZT treatment nor BDL affected the total protein expression of MAP2 and synaptophysin (Fig. $5 a-c$ ) in the rat cortex and hippocampus. The data from confocal laser scanning microscopy demonstrated that AZT treatment markedly decreased MAP2 pixel intensity in the cortex in BDL rats (Fig. $5 d$ ). These alterations 
a

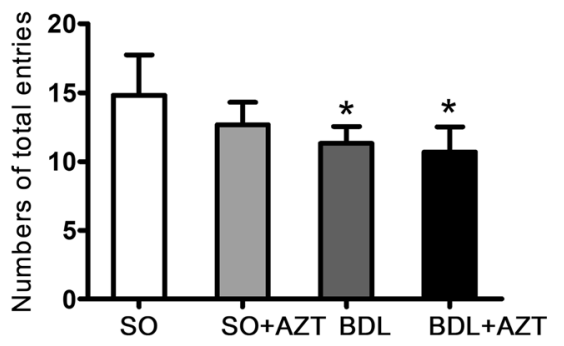

b

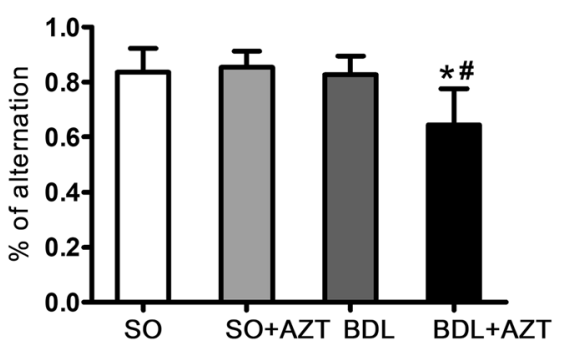

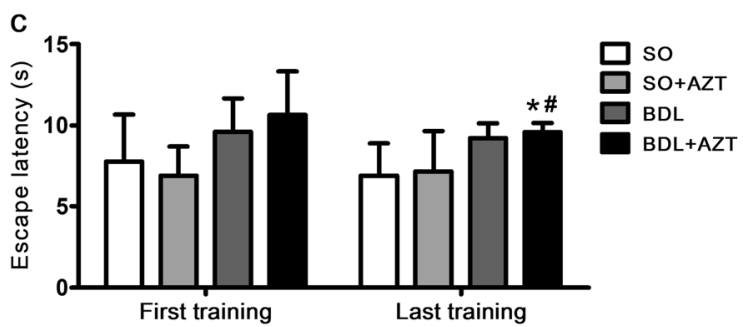

Fig. 3 Effects of zidovudine (AZT) treatment on the cognitive function (a, b) and exploratory ability (c) of sham-operated (SO) and bile duct ligation (BDL) rats. The data are expressed as the mean \pm SD for five rats. ${ }^{*} P<0.05$ vs SO rats and ${ }^{\#} P<0.05$ vs $S O+A Z T$ rats
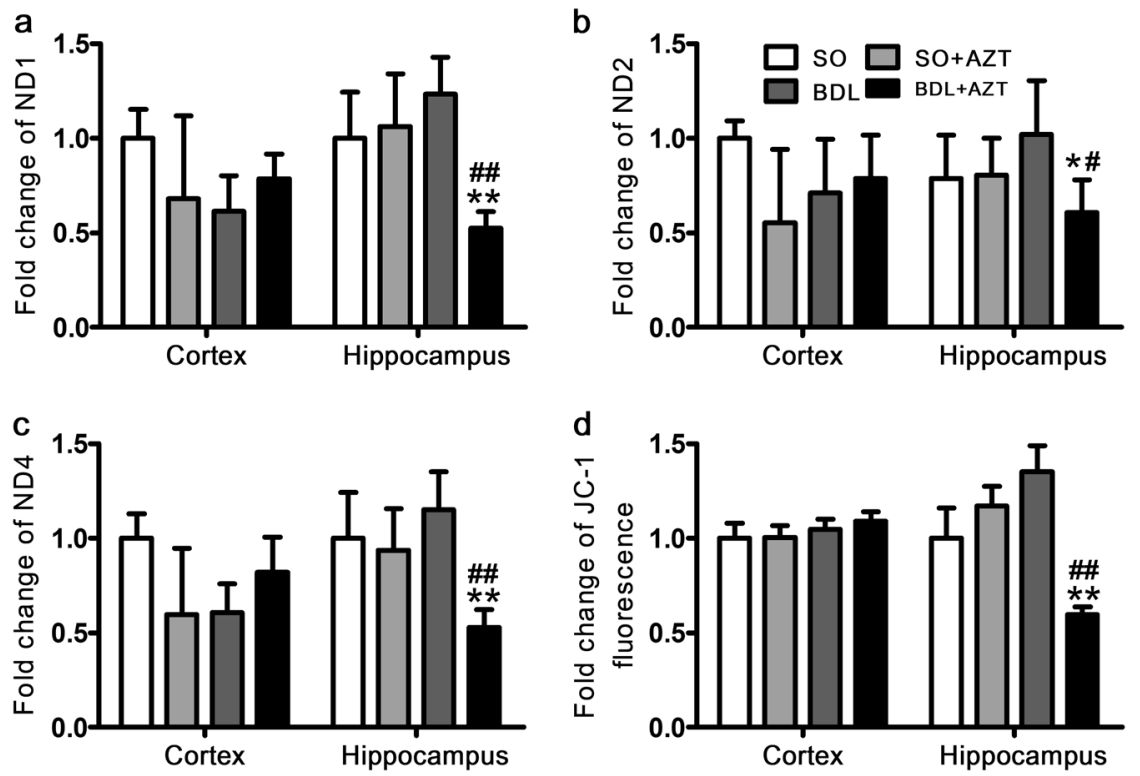

Fig. 4 Effects of zidovudine (AZT) on mitochondrial DNA (ND1, ND2, and ND4) levels (a-c) and mitochondrial membrane potential (d) in the brain tissues of sham-operated (SO) and bile duct ligation (BDL) rats. The data are expressed as the mean \pm SD for five rats. ${ }^{*} P<0.05$ and ${ }^{* *} P<$ 0.01 vs SO rats; ${ }^{\#} P<0.05$ and ${ }^{\# \#} P<0.01$ vs BDL rats

were not observed in the rat hippocampus. The hippocampal synaptophysin pixel intensities in BDL + AZT and BDL rats were lower than those in $\mathrm{SO}$ rats and $\mathrm{SO}+\mathrm{AZT}$ rats (Fig. 5e-f).

Uptake of AZT in the BBB and in MDCKII-BCRP cells The uptake of AZT in MDCKII-WT, MDCKII-BCRP, and HCMEC/D3 cells was measured. The results showed that the uptake of AZT in MDCKII-BCRP cells was markedly decreased to only approximately $50.0 \%$ of that in MDCKII-WT cells (Fig. $6 a$, b), further confirming that BCRP mediated the transport of AZT. It was found that coincubation with a BCRP inhibitor (Ko143) markedly increased the uptake of AZT in HCMEC/D3 cells (Fig. 6c), which constitute in vitro BBB cell model. The roles of BCRP in the transport of AZT were further confirmed by siRNA knockdown. The expression of BCRP decreased markedly to $27 \%$ or $19 \%$ of CON levels after knockdown for 48 or $60 \mathrm{~h}$, respectively, demonstrating successful silencing of BCRP (Fig. 6d, e). The uptake of AZT was significantly increased in the siRNA group (Fig. 6f), confirming that BCRP was involved in the transport of AZT in HCMEC/D3 cells. Incubation with serum of liver failure rats or with Ko143 markedly increased the uptake of AZT in MDCKII-BCRP cells but did not affect the uptake of AZT in wild-type MDCKII cells (Fig. 6g). High levels of bilirubin, especially unconjugated bilirubin, have been measured in BDL rats [16]. Our previous study showed that coincubation with unconjugated bilirubin impaired the expression and function of BCRP in both MDCKII-BCRP and HCMEC/D3 cells. Consistent with this finding, unconjugated bilirubin concentration-dependently increased the uptake of AZT in MDCKIIBCRP cells (Fig. 6h), suggesting that unconjugated bilirubin increased the uptake of AZT partly by impairing the expression and function of BCRP. 
a

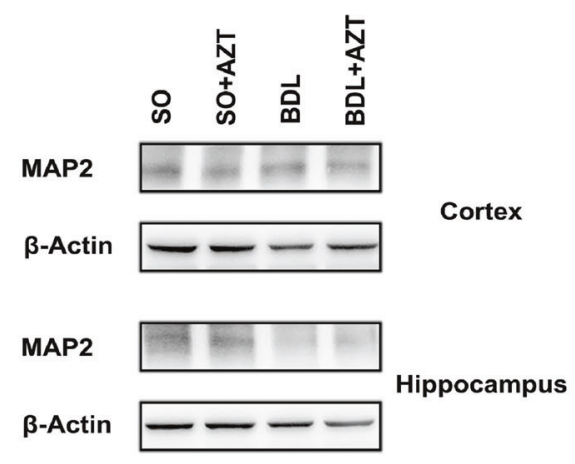

b

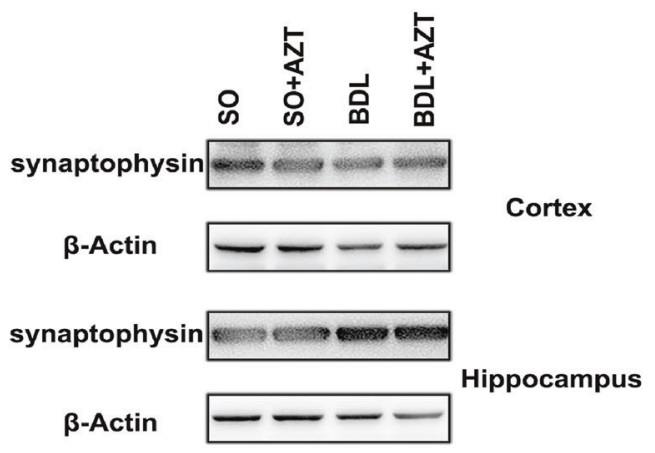

C

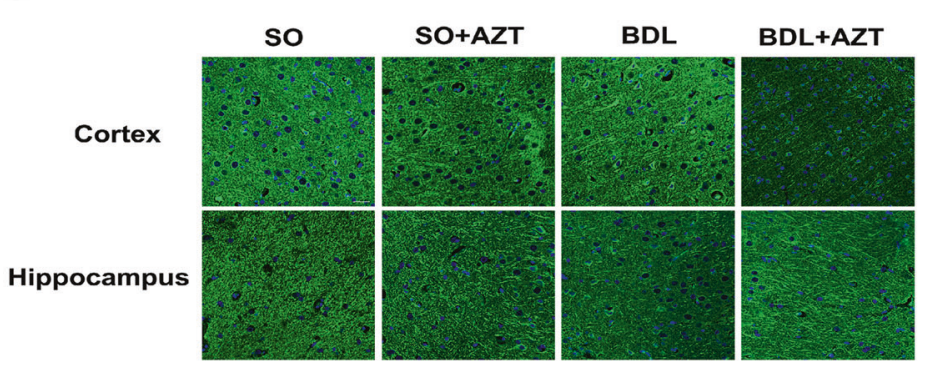

e

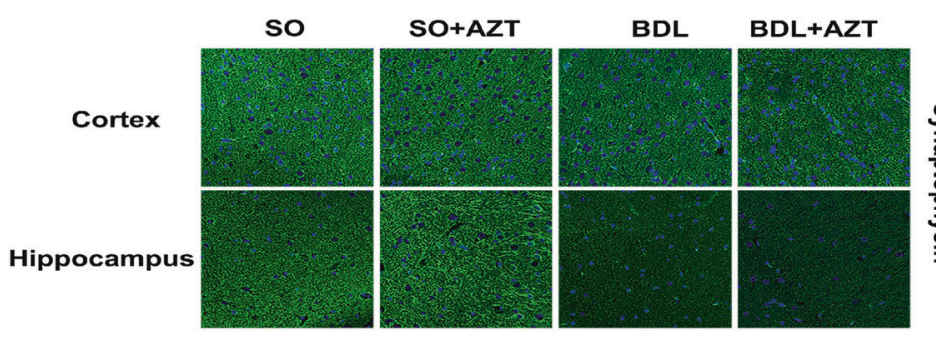

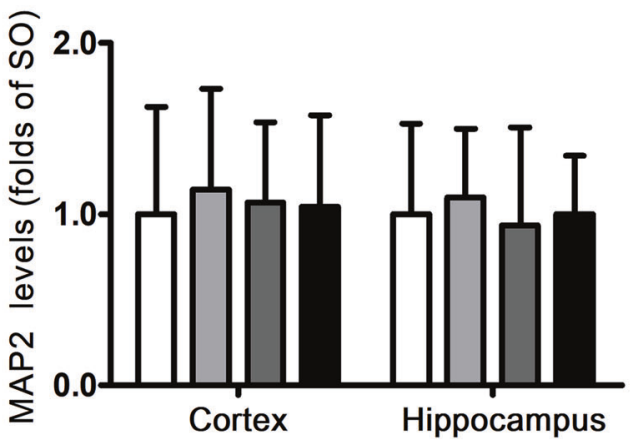

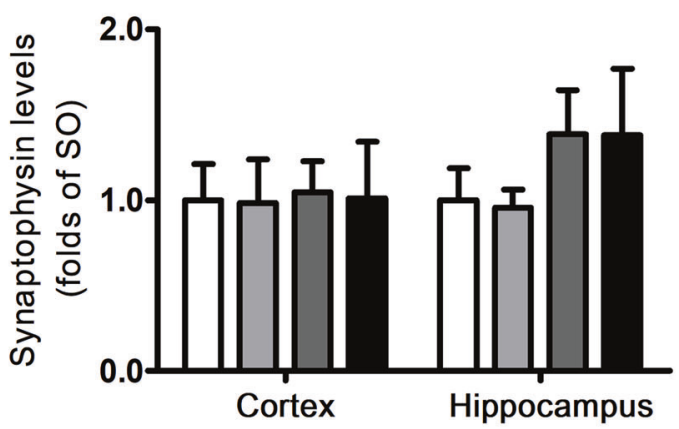

d
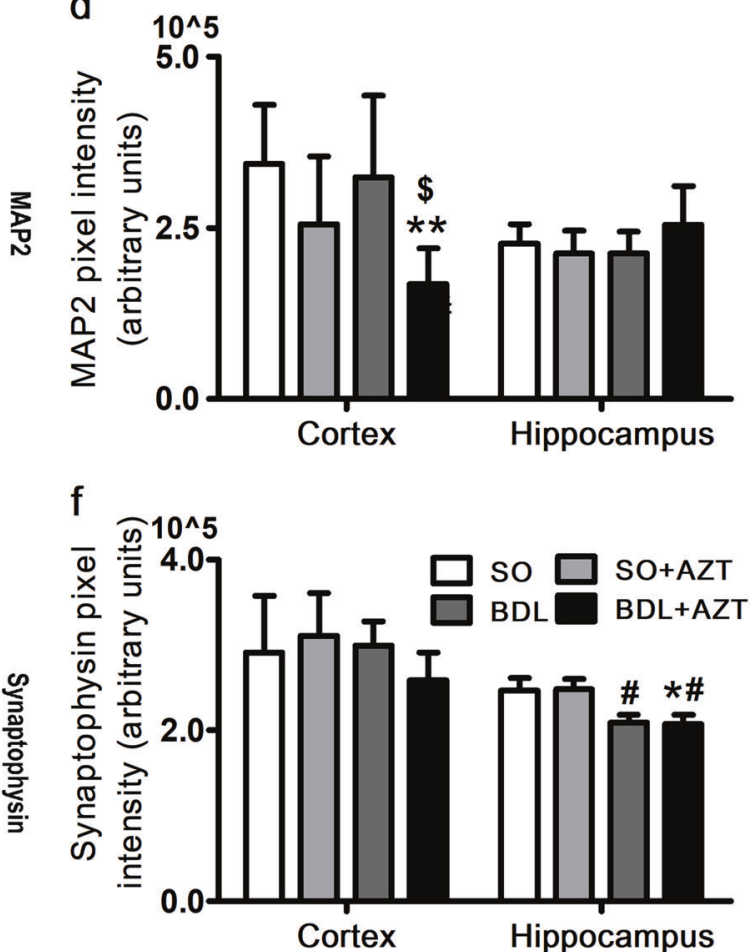

Fig. 5 Effects of zidovudine (AZT) treatment on the protein expression of microtubule-associated 2 (MAP2) (a) and synaptophysin (b) in the brains of sham-operated (SO) and bile duct ligation (BDL) rats. The data are expressed as the mean \pm SD for six rats. MAP2 (c, d) and synaptophysin (e, f) pixel intensity (AF488 stain, $\times 20$ ) in the rat hippocampus and cortex, as assessed using confocal microscopy. The data are expressed as the mean \pm SD for five rats. ${ }^{*} P<0.05$ vs SO rats; ${ }^{\#} P<0.05$ vs $\mathrm{SO}+\mathrm{AZT}$ rats; ${ }^{\$} P<0.05$ vs $\mathrm{BDL}$ rats

\section{DISCUSSION}

AZT, a typical nucleoside/nucleotide reverse transcriptase inhibitor, is widely used in the treatment of AIDS to inhibit viral RNAdependent DNA polymerase. Moreover, AZT inhibits mitochondrial polymerase $\gamma$, which is an enzyme required to maintain mtDNA, leading to mtDNA decreases, mitochondrial membrane potential loss, oxidative phosphorylation, and neuronal presynaptic terminal induction and ultimately causing impaired neurogenesis [24, 28]. In addition, 3'-amino-3'-deoxythymidine (AMT), a highly toxic catabolite of AZT, is involved in cytostatic and cytotoxic effects. Enhanced AMT levels in plasma affect the pharmacodynamic properties of AZT, increasing AZT activity against HIV replication and its cytotoxicity [29]. Studies have demonstrated that AMT is 5- to 7-fold more toxic to human 
a

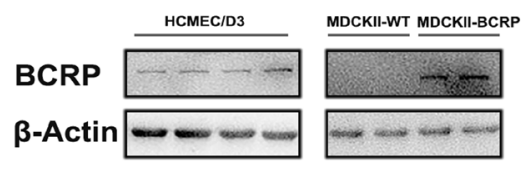

d

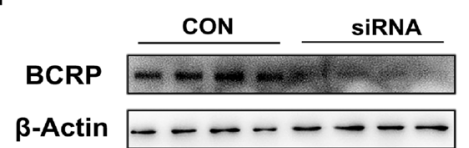

$48 \mathrm{~h}$

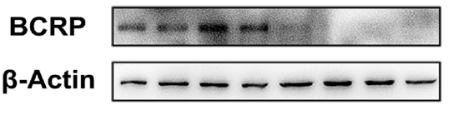

$60 \mathrm{~h}$ b
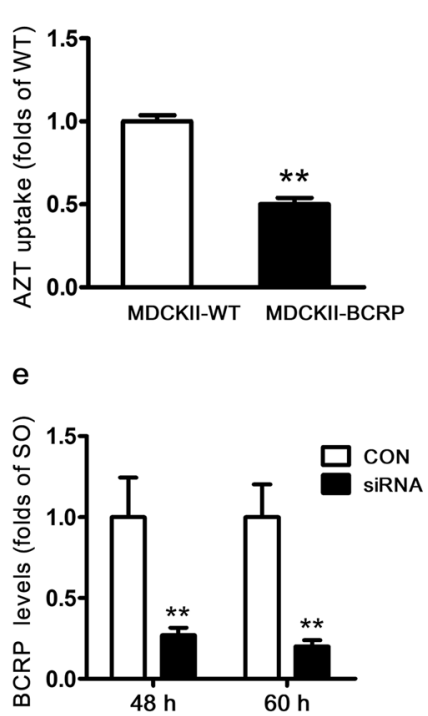
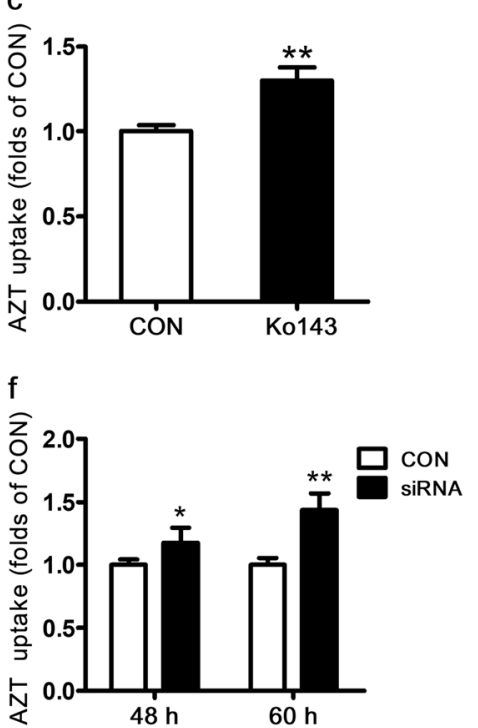

$\mathrm{g}$

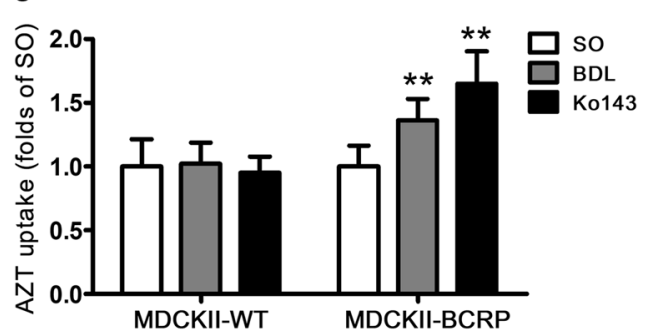

$\mathrm{h}$

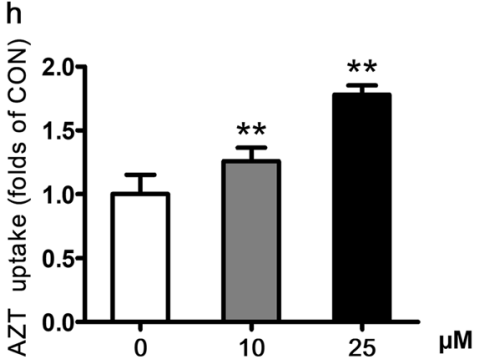

Fig. 6 Uptake of zidovudine (AZT) in MDCKII and HCMEC/D3 cells. Expression of BCRP in MDCKII-WT, MDCKII-BCRP, and HCMEC/D3 cells (a). The AZT concentrations in MDCKII-WT and MDCKII-BCRP cells (b) and in HCMEC/D3 cells with or without Ko143 (c) are shown. Effects of BCRP knockdown on BCRP expression and AZT uptake in HCMEC/D3 cells (d-f). AZT concentrations in MDCKII-WT and MDCKII-BCRP cells cocultured with serum of liver failure rats or with Ko143 $(\mathbf{g})$. Effects of unconjugated bilirubin on the uptake of AZT in MDCKII-BCRP cells (h). The data are expressed as the mean \pm SD for four cells. ${ }^{*} P<0.05$ and ${ }^{*} P<0.01$ vs CON

granulocyte-macrophage colony-forming units and erythroid burst-forming units than AZT in human hematopoietic progenitor cells [30]. Under normal conditions, the existence of the BBB limits the penetration of AZT into the brain [7, 11, 31]; thus, CNS toxicity of $A Z T$ is minimal or uncommon. Although the plasma protein binding of AZT is only $10 \%$ [32], AZT is a substrate of BCRP [33], which may be one of the reasons for the low penetration of AZT. We previously reported that BDL markedly impairs the function and expression of BCRP in rats [16], indicating that impairment of $\mathrm{BCRP}$ function and expression in the brain by BDL might increase the penetration of AZT into the brain, leading to increased exposure and neurotoxicity of AZT.

We first investigated alterations in the brain distribution of AZT in $\mathrm{BDL}$ rats. The results showed that the concentrations of $A Z T$ in brain homogenates of SO rats were markedly lower than those in plasma and that the brain homogenate-to-plasma AUC ratios were only 0.04 , close to the value reported previously for mice $(0.064)$ [34], which demonstrated the low penetration of AZT into the brain. The brain concentrations of AZT in BDL rats were 1.6-fold higher than those in SO rats, consistent with the increase in the brain distribution of prazosin (BCRP substrate). Moreover, the $t_{1 / 2}$ values of $A Z T$ in the brains of $B D L$ rats were longer than those in the brains of $\mathrm{SO}$ rats, while the $t_{1 / 2}$ values of AZT in plasma were identical between $B D L$ and $S O$ rats; these findings indicate that elimination of AZT from the brain was slowed down in BDL rats, possibly due to a decrease in the efflux of AZT from the brain. AZT was once reported to be a substrate of BCRP [8]. The data from both MDCKII-BCRP and HCMEC/D3 cells (an in vitro BBB model) also demonstrated that BCRP mediated the efflux of AZT at the $B B B$, which was further confirmed by silencing of the BCRP gene. It was found that coadministration of Ko143 (a BCRP inhibitor) markedly increased the influx of AZT into the hippocampus in normal rats. All these results suggested that the increased brain distribution of $A Z T$ in $B D L$ rats was at least partly attributable to the impaired function and expression of brain BCRP.

Next, we investigated whether increased brain distribution of AZT enhanced CNS toxicity, which was characterized by assessment of cognitive function. Cognitive function was assessed using Y-maze and two-step shuttle box trials. The data from the Y-maze trial showed that AZT treatment impaired cognitive function in $\mathrm{BDL}$ rats, resulting in a lower number of total entries and a lower percentage of alternations in arm entrance, suggesting impairment of the willingness of the rats to explore new environments. In the two-step shuttle box trials, it was found that AZT treatment distorted memory retention in BDL rats, as evidenced by a decrease in step-through latency time. These alterations were not observed in SO rats treated with AZT, suggesting that the memory injury in BDL rats was associated with AZT treatment and was partly attributable to the increased brain distribution of AZT. As described above, the increased brain distribution of AZT was partly due to a decrease in the expression and function of brain BBB proteins. Several animal experiments have demonstrated that the expression of BCRP in the brain is significantly increased during development [35], which might explain why abnormal mitochondrial respiratory chain complex activity, brain morphological alternations, neurological anomalies, cognitive function 
impairments, and seizures have been reported in children exposed to AZT in utero and after birth [36, 37].

In general, the toxicity of AZT often involves impairment of mitochondrial function. Mitochondrial function was assessed by evaluating mtDNA (ND1, ND2, and ND4) copy numbers and mitochondrial membrane potential. The results indicated that AZT treatment decreased the copy numbers of ND1, ND2, and ND4 in the hippocampus in BDL rats. AZT treatment in BDL rats decreased the hippocampal mitochondrial membrane potential to $60 \%$ of that in non-AZT-treated BDL rats. No alteration was found in the cortex in BDL rats. These observations indicated that AZT mainly impaired mitochondrial function in the hippocampus in BDL rats. It has been reported that antiretrovirals can cause neuronal damage and alter MAP2 and synaptophysin, which are often used to characterize neuronal injury $[24,38]$. Our findings showed that although the total protein expression levels of MAP2 and synaptophysin were comparable among the tested rats, confocal laser scanning microscopy analysis showed that AZT treatment markedly decreased MAP2 pixel intensity in cortices from BDL rats. A statistically significant decrease in synaptophysin pixel intensity was also found in hippocampi from BDL rats and BDL + AZT rats compared to SO and SO + AZT rats, demonstrating impairment of neuronal function. It has been reported that the hippocampus plays a prominent role in cognitive damage [39]. The present study demonstrated that mitochondrial function and neuronal synaptic activity were more easily impaired in the hippocampus than in the cortex.

In addition to BCRP, other transporters, such as P-GP [40] and organic anion transporter 3 [41], are also involved in the transport of AZT across the BBB. Our preliminary experiments showed that BDL increased rather than decreased the function of P-GP in the brains of rats (data not shown). In vitro data showed that a typical inhibitor of P-GP, verapamil $(100 \mu \mathrm{M})$, could not affect AZT uptake in either MDCKII-P-GP cells or HCMEC/D3 cells $[42,43]$, indicating that the contribution of P-GP to alterations in the brain distribution of AZT in $B D L$ rats requires further investigation. Liver failure impairs $B C R P$ function, potentially increasing the amount of AZT in the brains of patients and in turn increasing the risk of CNS toxicity. From this point of view, patients with liver failure need a more thorough knowledge of how to prevent CNS side effects during AZT treatment. Moreover, clinical reports have shown that impairment of liver function increases plasma AZT exposure. Patients undergoing AZT-based regimens such as $A Z T+3 T C+N V P$ and AZT + 3TC + EFZ present with the majority of ADRs, such as anemia (up to $36 \%$ ) and CNS side effects (up to $33 \%$ ) [44]. EFZ has been reported to inhibit the function of BCRP [45], indicating that increases in AZT-induced adverse CNS effects might be partly attributable to the inhibition of BCRP function by EFZ. Several studies have demonstrated that BCRP also mediates the efflux of other antiretrovirals $[40,42,43]$ such as lamivudine and saquinavir, indicating that the neurotoxicity of these agents in the CNS could be enhanced under liver failure due to impairment of BCRP function and expression.

In conclusion, decreased function and expression of BCRP in $B D L$ rats increased the brain distribution of $A Z T$. The increased brain distribution of AZT in turn enhanced the CNS toxicity of AZT and ultimately led to mitochondrial dysfunction, neuronal damage, and cognitive dysfunction.

\section{ACKNOWLEDGEMENTS}

This study was supported by the National Natural Science Foundation of China (Nos. 81573490,81872930 , and 81673505), the Natural Science Foundation of Jiangsu Province (BK20161457), and the "Double First-Class" University Project (CPU2018GY22).

\section{AUTHOR CONTRIBUTIONS}

$Y Y Q, L L$, and $X D L$ designed the experiments, analyzed the data, and wrote the manuscript; YYQ, PX, TW, CQQ, YLF, DHG, LZ, WMK, HYY, and YTY conducted experiments; YYQ performed data analysis; and FX, XDL, and LL. reviewed the manuscript.

\section{ADDITIONAL INFORMATION}

Conflict of interest: The authors declare that they have no conflict of interest.

\section{REFERENCES}

1. Chow WA, Jiang C, Guan M. Anti-HIV drugs for cancer therapeutics: back to the future? Lancet Oncol. 2009;10:61-71.

2. Abers MS, Shandera WX, Kass JS. Neurological and psychiatric adverse effects of antiretroviral drugs. CNS Drugs. 2014;28:131-45.

3. Scruggs ER, Naylor AJD. Mechanisms of zidovudine-induced mitochondrial toxicity and myopathy. Pharmacology. 2008;82:83-8.

4. Kakuda TN. Pharmacology of nucleoside and nucleotide reverse transcriptase inhibitor-induced mitochondrial toxicity. Clin Ther. 2000;22:685-708.

5. Lewis W, Day BJ, Copeland WC. Mitochondrial toxicity of NRTI antiviral drugs: an integrated cellular perspective. Nat Rev Drug Discov. 2003;2:812-22.

6. Kohler JJ, Lewis W. A brief overview of mechanisms of mitochondrial toxicity from NRTIs. Environ Mol Mutagen. 2007;48:166-72.

7. Marraa CM, Zhao Y, Clifford DB, Letendre S, Evans S, Henry K. et al. Study ACTG. Impact of combination antiretroviral therapy on cerebrospinal fluid HIV RNA and neurocognitive performance. Aids. 2009;23:1359-66.

8. Pan GY, Giri N, Elmquist WF. Abcg2/Bcrp1 mediates the polarized transport of antiretroviral nucleosides abacavir and zidovudine. Drug Metab Dispos. 2007;35:1165-73.

9. Wang $Y Z$, Duan $X L$, Li YF, Liang $X J$. Multidrug resistance mediated by half $A B C$ transporter ABCG2. Prog Biochem Biophys. 2009;36:1523-9.

10. Wu DA, Clement JG, Pardridge WM. Low blood-brain barrier permeability to azidothymidine (AZT), 3TC (TM) and thymidine in the rat. Brain Res. 1998;791:313-6.

11. Fox E, Bungay PM, Bacher J, McCully $C L$, Dedrick RL, Balis FM. Zidovudine concentration in brain extracellular fluid measured by microdialysis: steadystate and transient results in rhesus monkey. J Pharmacol Exp Ther. 2002;301:1003-11.

12. Giri N, Shaik N, Pan GY, Terasaki T, Mukai C, Kitagaki S, et al. Investigation of the role of breast cancer resistance protein (Bcrp/Abcg2) on pharmacokinetics and central nervous system penetration of abacavir and zidovudine in the mouse. Drug Metab Dispos. 2008;36:1476-84.

13. Filia MF, Marchini T, Minoia JM, Roma MI, De Fino FT, Rubio MC, et al. Induction of $A B C G 2 / B C R P$ restricts the distribution of zidovudine to the fetal brain in rats. Toxicol Appl Pharmacol. 2017;330:74-83.

14. Jin S, Wang XT, Liu L, Yao D, Liu C, Zhang M, et al. P-glycoprotein and multidrug resistance-associated protein 2 are oppositely altered in brain of rats with thioacetamide-induced acute liver failure. Liver Int. 2013;33:274-82.

15. Liu L, Miao MX, Chen Y, Wang ZJ, Sun BB, Liu XD. Altered function and expression of $A B C$ transporters at the blood-brain barrier and increased brain distribution of phenobarbital in acute liver failure mice. Front Pharmacol. 2018;9:190.

16. Xu P, Ling ZL, Zhang J, Li Y, Shu N, Zhong ZY, et al. Unconjugated bilirubin elevation impairs the function and expression of breast cancer resistance protein (BCRP) at the blood-brain barrier in bile duct-ligated rats. Acta Pharmacol Sin. 2016;37:1129-40.

17. Brown MW, Aggleton JP. Recognition memory: what are the roles of the perirhinal cortex and hippocampus? Nat Rev Neurosci. 2001;2:51-61.

18. Chen LJ, Wang YJ, Chen JR, Tseng GF. Hydrocephalus compacted cortex and hippocampus and altered their output neurons in association with spatial learning and memory deficits in rats. Brain Pathol. 2017;27:419-36.

19. Fernández G, Effern A, Grunwald T, Pezer N, Lehnertz K, Dümpelmann $M$, et al. Real-time tracking of memory formation in the human rhinal cortex and hippocampus. Science. 1999;285:1582-5.

20. Zhou HH, Jin CL, Cui LL, Xing HJ, Liu J, Liao W, et al. HMGB1 contributes to the irradiation-induced endothelial barrier injury through receptor for advanced glycation endproducts (RAGE). J Cell Physiol. 2018;233:6714-21.

21. Triguero D, Buciak J, Pardridge WM. Capillary depletion method for quantification of blood-brain barrier transport of circulating peptides and plasma proteins. J Neurochem. 2010;54:1882-8.

22. Liu XD, Liu GQ. P-glycoprotein regulated transport of glutamate at blood-brain barrier. Acta Pharmacol Sin. 2001;22:111-6.

23. Alata W, Paris-Robidas S, Emond V, Bourasset F, Calon F. Brain uptake of a fluorescent vector targeting the transferrin receptor: a novel application of in situ brain perfusion. Mol Pharm. 2014;11:243-53.

24. Akay C, Cooper M, Odeleye A, Jensen BK, White MG, Vassoler F, et al. Antiretroviral drugs induce oxidative stress and neuronal damage in the central nervous system. J Neurovirol. 2014;20:39-53. 
25. Martini M, Calandreau L, Jouhanneau M, Mhaouty-Kodja S, Keller M. Perinatal exposure to methoxychlor enhances adult cognitive responses and hippocampal neurogenesis in mice. Front Behav Neurosci. 2014;8:202.

26. Abdel-Aziz AK, Mantawy EM, Said RS, Helwa R. The tyrosine kinase inhibitor, sunitinib malate, induces cognitive impairment in vivo via dysregulating VEGFR signaling, apoptotic and autophagic machineries. Exp Neurol. 2016;283(Pt A):129-41.

27. Yin L, Castagnino P, Assoian RK. ABCG2 expression and side population abundance regulated by a transforming growth factor beta-directed epithelialmesenchymal transition. Cancer Res. 2008;68:800-7.

28. Nolan D, Mallal S. Complications associated with NRTI therapy: update on clinical features, and possible pathogenic mechanisms. Antivir Ther. 2004;9:849-63.

29. Cretton EM, Schinazi RF, Mcclure HM, Anderson DC, Sommadossi JP. Pharmacokinetics of 3'-azido-3'-deoxythymidine and its catabolites and interactions with probenecid in rhesus monkeys. Antimicrob Agents Chemother. 1991;35:801-7.

30. Cretton EM, Xie MY, Bevan RJ, Goudgaon NM, Schinazi RF, Sommadossi JP. Catabolism of $3^{\prime}$-azido-3'-deoxythymidine in hepatocytes and liver microsomes, with evidence of formation of 3'-amino-3'-deoxythymidine, a highly toxic catabolite for human bone marrow cells. Mol Pharmacol. 1991;39:258-66.

31. Galinsky RE, Hoesterey BL, Anderson BD. Brain and cerebrospinal fluid uptake of zidovudine $(A Z T)$ in rats after intravenous injection. Life Sci. 1990;47:781-8.

32. Stahle L, Guzenda E, Ljungdahlstahle E. Pharmacokinetics and extracellular distribution to blood, brain, and muscle of alovudine (3'-fluorothymidine) and zidovudine in the rat studied by microdialysis. J Acquir Immune Defic Syndr Hum Retrovirol. 1993;6:435-9.

33. Xin W, Tatsuhiko F, Takao N, Mika O, Yoshikazu S, Shin-Ichi A, et al. Breast cancer resistance protein (BCRP/ABCG2) induces cellular resistance to HIV-1 nucleoside reverse transcriptase inhibitors. Antiviral Chem Chemother. 2005;16:213.
34. Doshi KJ, Gallo JM, Boudinot FD, Schinazi RF, Chu CK. Comparative pharmacokinetics of 3'-azido-3'-deoxythymidine (AZT) and 3'-azido-2',3'-dideoxyuridine (AZddU) in mice. Drug Metab Dispos Biol Fate Chem. 1989;17:590-4.

35. Soares RV, Do TM, Mabondzo A, Pons G, Chhun S. Ontogeny of ABC and SLC transporters in the microvessels of developing rat brain. Fundam Clin Pharmacol. 2016;30:107-16.

36. Newschaffer CJ, Cocroft J, Anderson CE, Hauck WW, Turner BJ. Prenatal zidovudine use and congenital anomalies in a Medicaid population. J Acquir Immune Defic Syndr. 2000;24:249-56.

37. Blanche S, Tardieu M, Rustin P, Slama A, Barret B, Firtion G, et al. Persistent mitochondrial dysfunction and perinatal exposure to antiretroviral nucleoside analogues. Lancet. 1999;354:1084-9.

38. Sanchez AB, Kaul M. Neuronal stress and injury caused by HIV-1, CART and drug abuse: converging contributions to HAND. Brain Sci. 2017;7:25.

39. Memory BO. Function and the hippocampus. Monogr Neural Sci. 2014;34:51-59.

40. De Souza J, Benet LZ, Huang Y, Storpirtis S. Comparison of bidirectional lamivudine and zidovudine transport using MDCK, MDCK-MDR1, and Caco-2 cell monolayers. J Pharm Sci. 2009;98:4413-9.

41. Gibbs JE, Thomas SA. The distribution of the anti-HIV drug, 2' $3^{\prime}$-dideoxycytidine (ddC), across the blood-brain and blood-cerebrospinal fluid barriers and the influence of organic anion transport inhibitors. J Neurochem. 2002;80:392-404.

42. Wang $X$, Nitanda T, Shi MY, Okamoto M, Furukawa T, Sugimoto $Y$, et al. Induction of cellular resistance to nucleoside reverse transcriptase inhibitors by the wildtype breast cancer resistance protein. Biochem Pharmacol. 2004;68:1363-70.

43. Janneh O, Owen A, Chandler B, Hartkoorn RC, Hart CA, Bray PG, et al. Modulation of the intracellular accumulation of saquinavir in peripheral blood mononuclear cells by inhibitors of MRP1, MRP2, P-gp and BCRP. Aids. 2005;19:2097-102.

44. Mania Z. Zidovudine related mania. Med J Aust. 1989;150:339-41.

45. Johanna W, Johanna R, Caroline Henrike S, Nahal KK, Alexandra S, Walter Emil H, et al. Modulation of human BCRP (ABCG2) activity by anti-HIV drugs. J Antimicrob Chemother. 2007:59:238-45. 